\title{
A protocol for the culture and isolation of murine synovial fibroblasts
}

\author{
JINJUN ZHAO ${ }^{1 *}$, QINGQING OUYANG ${ }^{1 *}$, ZIYOU $\mathrm{HU}^{2}$, \\ QIN HUANG ${ }^{1}$, JING WU $^{1}$, RAN WANG $^{1}$ and MIN YANG ${ }^{1}$ \\ ${ }^{1}$ Department of Rheumatology and Immunology; ${ }^{2}$ Research Center of Clinical Medicine, \\ Nanfang Hospital, Guangzhou, Guangdong 510515, P.R. China
}

Received March 15, 2016; Accepted May 24, 2016

DOI: $10.3892 /$ br.2016.708

\begin{abstract}
The culture of synovial fibroblasts (SFs) is one of the most effective tools for investigating the pathology and physiology of synovial tissues and should prove useful for identifying the importance of SFs in disease as well as for the development of novel therapeutic approaches for several chronic joint diseases, such as rheumatoid arthritis. However, thus far, a detailed protocol for the primary culture and isolation of murine SFs has not been established. Therefore, the present study describes an easy and convenient method for isolating and culturing SFs from C57BL/6 mice. This protocol can be divided into 4 stages: Isolation of synovial tissues, isolation of SFs, seeding of SFs for growth in culture and purity analysis of SFs using the four cell markers, vimentin, cluster of differentiation 90.2 (CD90.2; Thy-1.2), intracellular adhesion molecule 1 (CD54) and vascular cell adhesion molecule 1 (CD106). This method is efficient and a purified population of SFs can be obtained 10 days after the initiation of culture.
\end{abstract}

\section{Introduction}

Rheumatoid arthritis (RA) is a systemic autoimmune disease that is characterized by chronic invasive arthritis. The major pathological changes that occur during RA include synovitis and the resulting articular cartilage and bone damage, which ultimately lead to articular deformation. Without systematic treatment, RA will develop into a disability within 10 years in $\sim 45 \%$ of patients (1). Although the pathogenesis of RA has not been fully elucidated, it has been widely accepted that

Correspondence to: Professor Min Yang, Department of Rheumatology and Immunology, Nanfang Hospital, 1838 North Guangzhou Avenue, Guangzhou, Guangdong 510515, P.R. China E-mail: minyanggz@163.com

${ }^{*}$ Contributed equally

Key words: synovial fibroblasts, cell culture, C57BL/6 mouse, rheumatoid arthritis, isolation synovial fibroblasts (SFs) have an important role in the loss of cartilage tissue integrity (2-4). In healthy joints, the synovial lining at the border to the joint cavity consists of 1-3 cell layers, predominantly containing SFs and macrophages. The physiological functions of SFs are to provide the joint cavity and the adjacent cartilage with plasma protein and hyaluronic acid as a lubricant. SFs also produce matrix components, such as collagen and a series of matrix-degrading enzymes, to participate in the continuous matrix remodeling $(5,6)$. However, in RA, the synovial lining thickness increases to $10-15$ cell layers $(7,8)$, and the SFs are activated to become RASFs, which damage the cartilage by producing inflammatory cell factors and causing matrix degradation (9). Therefore, an improved understanding of the characteristics of SFs will be important for identifying the contribution of this specific cell type to the pathogenesis of RA.

To obtain large quantities of SFs from mice in vitro has been challenging in fundamental studies of RA. As large rodents, such as rats (10) and rabbits (11), provide a rich source of synovial tissues, it is easier to culture SFs in vitro. However, mice have a small volume of intra-articular synovium tissues, and therefore, it is difficult to obtain large quantities of SFs. A primary culture system for mouse SFs has not been established and remains a challenge, thus increasing the difficulty associated with performing relevant studies and impeding research investigating articular diseases. In the present study, an improved culture method for SFs was established in which after 10 days of culturing, the cells retained their original characteristics of constitutive expression of vimentin (12), cluster of differentiation 90.2 (CD90.2) (13), intracellular adhesion molecule 1 (ICAM-1) (14) and vascular cell adhesion molecule 1 (VCAM-1) (15). This method provides a high-yield and pure SFs population.

\section{Materials and methods}

Animals and ethics statement. The 10-week-old C57BL/6 mice were purchased from the Animal Laboratory of Southern Medical University (Guangzhou, Guangdong, China; license no. SCXK 2011-0015). The study was performed in accordance with the 'Guide for the Care and Use of Laboratory Animals' published by the US National Institutes of Health (NIH, publication no. 85-23, revised 1996). The experiments 
were approved by the Institutional Animal Care and Use Committee of Southern Medical University.

Tools and reagents. Microsurgery scissors and forceps were purchased from Jiaxing Moore Trade Co. (Jiaxing, China), $0.2-\mu \mathrm{m}$ syringe filters were purchased from Jet Bio-filtration (Guangzhou, China), and 1.5-ml Eppendorf tubes, 60-mm Petri dishes, 3-ml plastic pipettes and $75-\mathrm{cm}^{2}$ flasks were purchased from Corning (Corning, NY, USA). Penicillin-streptomycin, Dulbecco's modified Eagle's medium (DMEM) and fetal bovine serum (FBS) were obtained from Gibco (Thermo Fisher Scientific, Inc., Waltham, MA, USA). Type IV collagenase, Tween-20, Triton X-100, phosphate-buffered saline (PBS) and bovine serum albumin (BSA) were purchased from Sigma-Aldrich (St. Louis, MO, USA). Purified rat anti-mouse CD106 (cat. no. 553330), purified rat immunoglobulin G2a (IgG2a), $\kappa$ isotype control (cat. no. 553927), FITC goat anti-rat Ig secondary antibody (cat. no. 554016), phycoerythrin (PE) hamster anti-mouse CD54 (cat. no. 553253), PE hamster IgG1 $\kappa$ isotype control (cat. no. 553972), biotin rat anti-mouse CD90.2 (cat. no. 553011), biotin rat IgG2b, $\kappa$ isotype control (cat. no. 553987) and PE-streptavidin (cat. no. 554061) were obtained from BD Pharmingen (Franklin Lakes, NJ, USA), vimentin rabbit monoclonal antibody (Alexa Flour 488 Conjugate; cat. no. 9854) was obtained from Cell Signaling Technology (Beverly, MA, USA).

Preparation of reagents. Culture medium was DMEM supplemented with $1 \%$ of penicillin-streptomycin and $10 \%$ of FBS. For $1 \%$ type IV collagenase, $100 \mathrm{mg}$ of type IV collagenase was reconstituted in $10 \mathrm{ml}$ of PBS, filter sterilized with a $0.22-\mu \mathrm{m}$ filter and $1 \mathrm{ml}$ aliquots were frozen at $-20^{\circ} \mathrm{C}$. For PBS with Tween-20 (PBST), $100 \mu \mathrm{l}$ of Tween-20 was diluted in $100 \mathrm{ml}$ of PBS $(1: 1,000)$ and stored at room temperature. This reagent should be used within 2 months or prepared immediately before use. For $2 \%$ BSA, $2 \mathrm{~g}$ of BSA was dissolved in $100 \mathrm{ml}$ of PBS.

Isolation of synovial tissues. Mice were sacrificed by cervical dislocation and immersed in $75 \%$ alcohol for 2 min for sterilization. Under a stereomicroscope (Olympus, Tokyo, Japan), the skin of the hind limbs was removed and the synovial tissues around the hip joints were obtained using microsurgery scissors and forceps (white sponge; Fig. 1A). During this procedure, attention was focused on eliminating the 'egg-yolk'-like yellow oval substance (insert in Fig. 1B). The synovium is transferred to a $60-\mathrm{mm}$ Petri dish containing $2 \mathrm{ml}$ of DMEM.

To obtain greater amounts of synovial tissues, the following procedures were implemented: Isolated the hind limbs (preserved all the muscle tissues and discarded foot and ankles) and placed in a 60-mm Petri dish containing $2 \mathrm{ml}$ of DMEM. Under a stereomicroscope, the muscle inside the popliteal fossa was cut open with microsurgery scissors and forceps to harvest the synovium (insert 2 in Fig. 1C). During this procedure, attention was focused on eliminating the 'egg-yolk'-like yellow oval substance (insert in Fig. 1D) in the middle of the synovium. Subsequently, the synovium was transferred to another $60-\mathrm{mm}$ Petri dish containing $2 \mathrm{ml}$ of DMEM. To harvest the intra-articular synovium, the articular cavity of the knee was cut open along both sides of the patella under a stereomicroscope (insert 3 in Fig. 1C), and isolated the intra-articular synovium carefully. Of note, the connective tissues around the synovium were carefully eliminated under a stereomicroscope.

Digestion of the synovium and culture of SFs. The synovium was transferred into a $1.5-\mathrm{ml}$ Eppendorf tube containing $0.5 \mathrm{ml}$ DMEM and $0.5 \mathrm{ml} 1 \%$ type IV collagenase and the tissues were separated into $1-\mathrm{mm}^{3}$ blocks with microsurgery scissors. Subsequently, the Eppendorf tube was incubated at a constant temperature of $37^{\circ} \mathrm{C}$ in an orbital shaker incubator (200 rpm) for $60 \mathrm{~min}$. Following completion of the incubation time, the sample was vortexed vigorously for $1.5 \mathrm{~min}$ to release the cells. The sample was centrifuged for $5 \mathrm{~min}$ at $300 \mathrm{x} \mathrm{g}$ and resuspended with DMEM supplemented with 10\% FBS and $1 \%$ penicillin-streptomycin. The cells were seeded cells into a $75-\mathrm{cm}^{2}$ flask and placed in a humidified tissue culture incubator $\left(37^{\circ} \mathrm{C}, 5 \% \mathrm{CO}_{2}\right)$. This procedure was performed on a clean bench.

Immunofluorescence staining of SFs. Immunofluorescence cytochemical staining was performed of passage $3 \mathrm{SFs}$ cultured on a 12-well plate with anti-vimentin antibody. The simplified process is as follows: The medium was discarded and the cells were washed twice with PBS. Subsequently, the cells were fixed in $4 \%$ paraformaldehyde for $10 \mathrm{~min}$ and soaked with $1.5 \mathrm{ml}$ of $0.2 \%$ Triton X-100 solution for $5 \mathrm{~min}$. Following this the samples were washed twice with PBST. Subsequently, 200-300 $\mu$ l goat serum blocking reagent was added at room temperature for 20-30 min. The cells were incubated with $1 \mathrm{ml}$ of Alexa fluor 488 vimentin antibody $(1: 200)$ overnight at $4{ }^{\circ} \mathrm{C}$ in the dark. The cells were washed 3 times for 3 min with PBST. Following this, $200 \mu \mathrm{l}$ of PBS was added to the cells and the samples were protected from light until their observation using a fluorescence microscope.

Flow cytometric analysis of passage 3 SFs. Four cell markers, vimentin, CD90.2, ICAM-1 (CD54) and VCAM-1 (CD106) were used for the purity analysis of SFs in accordance with the instructions. Simplified procedure: After 3 passages, the cells were trypsinized, washed twice with $2 \%$ BSA and resuspended in $1 \mathrm{ml}$ of $2 \%$ BSA at a density of $1 \times 10^{7}$ cells $/ \mathrm{ml}$. Subsequently, $100 \mu 1$ of the cell suspension was added to a 1.5-ml Eppendorf tube. Fluorescently conjugated antibodies (or purified or biotin-conjugated antibodies) were added to the samples and incubated for $40 \mathrm{~min}$ at room temperature in the dark. The cells were washed twice with $1 \mathrm{ml}$ of stain buffer (2\% BSA). For indirect immunofluorescence staining, the cells were incubated with an appropriately labeled secondary antibody in $100 \mu \mathrm{l}$ of stain buffer for $20 \mathrm{~min}$ at room temperature and washed twice with $2 \%$ BSA. The cells were resuspended in $0.5 \mathrm{ml}$ of stain buffer and analyzed by flow cytometry.

\section{Results}

Culture of isolated SFs. Adhesion of the SFs began on day 2. The medium was changed initially on day 3 (Fig. 2A) and following this every 3-4 days. Cells usually reached confluency 


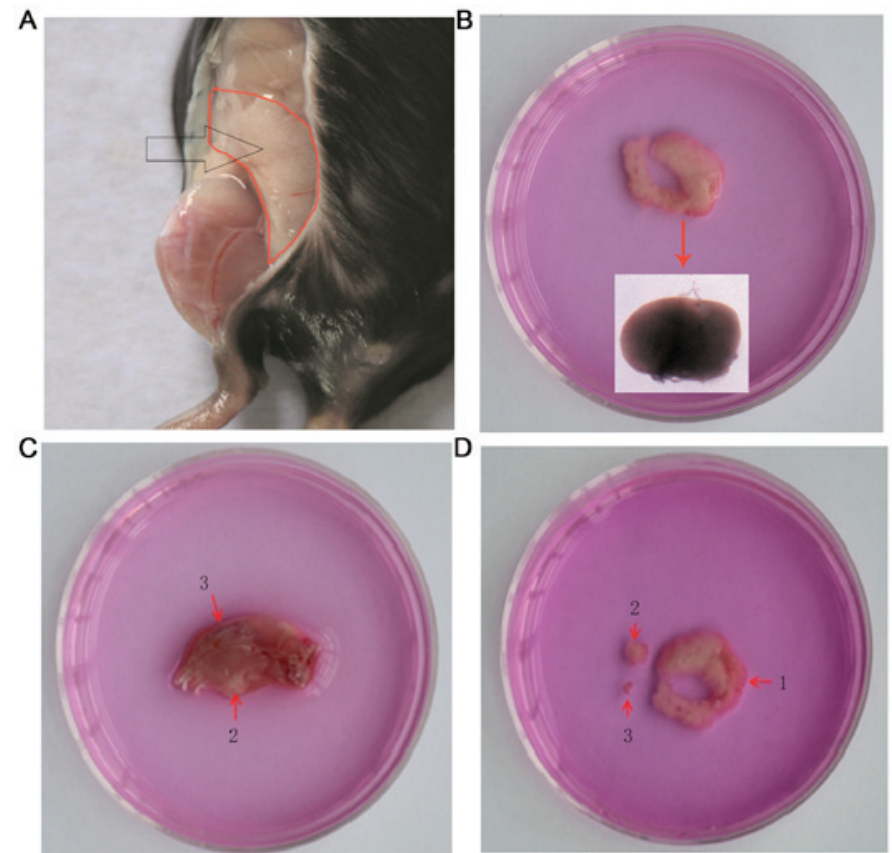

Figure 1. Isolation of synovial tissues from C57BL/6 mice. (A) The synovium around the hip joints. Under the stereomicroscope, the synovium appears white and spongy; however, the gross observation of the tissues shows a pink colour neighboring the peritoneum. (B) The dissociated synovium around the hip joints. At the turning of the specimen, there is a round substance that is similar to 'egg-yolk', as shown in the insert. The color of the image was distorted under the stereomicroscope; the actual color is yellow. (C) Dissociated hind limbs. 2, synovium is hidden in the deep popliteal fossa and was exposed by cutting open the muscle. The synovium obtained from this area contains a substance similar to 'egg-yolk' that is also found in the synovium around the hip joints. 3, synovial tissues of the knee joints, which were exposed by cutting the articular cavity of the knee open along the two sides of the patella. (D) Dissociated synovium. 1 , synovium around the hip joints; 2 , synovium in the popliteal fossa; 3 , synovium within the knee joints. The size of the synovium differs in the different location.
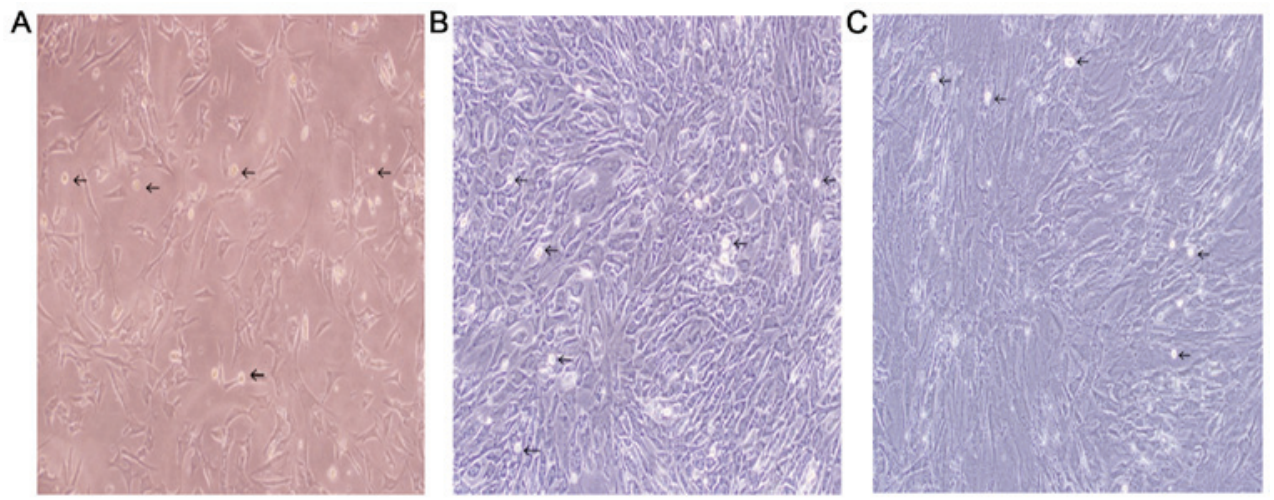

Figure 2. Culture of isolated SFs. (A) Micrographs of SFs plated on culture dishes after 3 days. The cells exhibited the typical spindle-like shape of fibroblasts, and some synovial macrophages were also observed. (B) Micrographs of SFs cultured after 6 days. Cells reached confluency 6 days after isolation. Due to the concentrated growth of the SFs, the number of synovial macrophages appeared to be limited. However, following digestion, a greater number of synovial macrophages was observed. (C) Micrographs of SFs cultured after 10 days. The number of synovial macrophages clearly decreased after passage 3 . The purity of the synovial fibroblasts increased significantly (arrows indicate macrophages). SFs, synovial fibroblasts.

6 days after isolation. After the first passage, confluency was reached 3-4 days later. The first passage was performed by trypsin digestion avoiding a long incubation with the enzyme (2-min incubation with pre-warmed trypsin is effective for fibroblast detachment, whereas macrophages that are highly adhesive remain attached. Thus, it is highly recommended to change culture flasks every passage). At this time, the number of synovial macrophages reached a maximum (Fig. 2B) and subsequently decreased with the passage number. After 3 passages (day 10; Fig. 2C), the synovial macrophages typically disappeared.
Purity analysis of SFs. Immunostaining of passage 3 SFs in primary cultures with an anti-vimentin antibody found that the majority of cells were positive (Fig. 3A and B). The flow cytometric analysis indicated that vimentin and CD90.2 labeled $>95 \%$ of cells (Fig. 3C and D). The purity of the CD54-labeled cells was $\sim 80 \%$ and the purity of the CD106-labeled cells was only $23 \%$ (Fig. 3E and F). The cause of the low purity of CD106-labeled cells remains to be elucidated. The present results demonstrated that the method described herein was simple and effective and can be used to obtain a large quantity of SFs $\left(0.75-1 \times 10^{7}\right.$ cells per flask). 

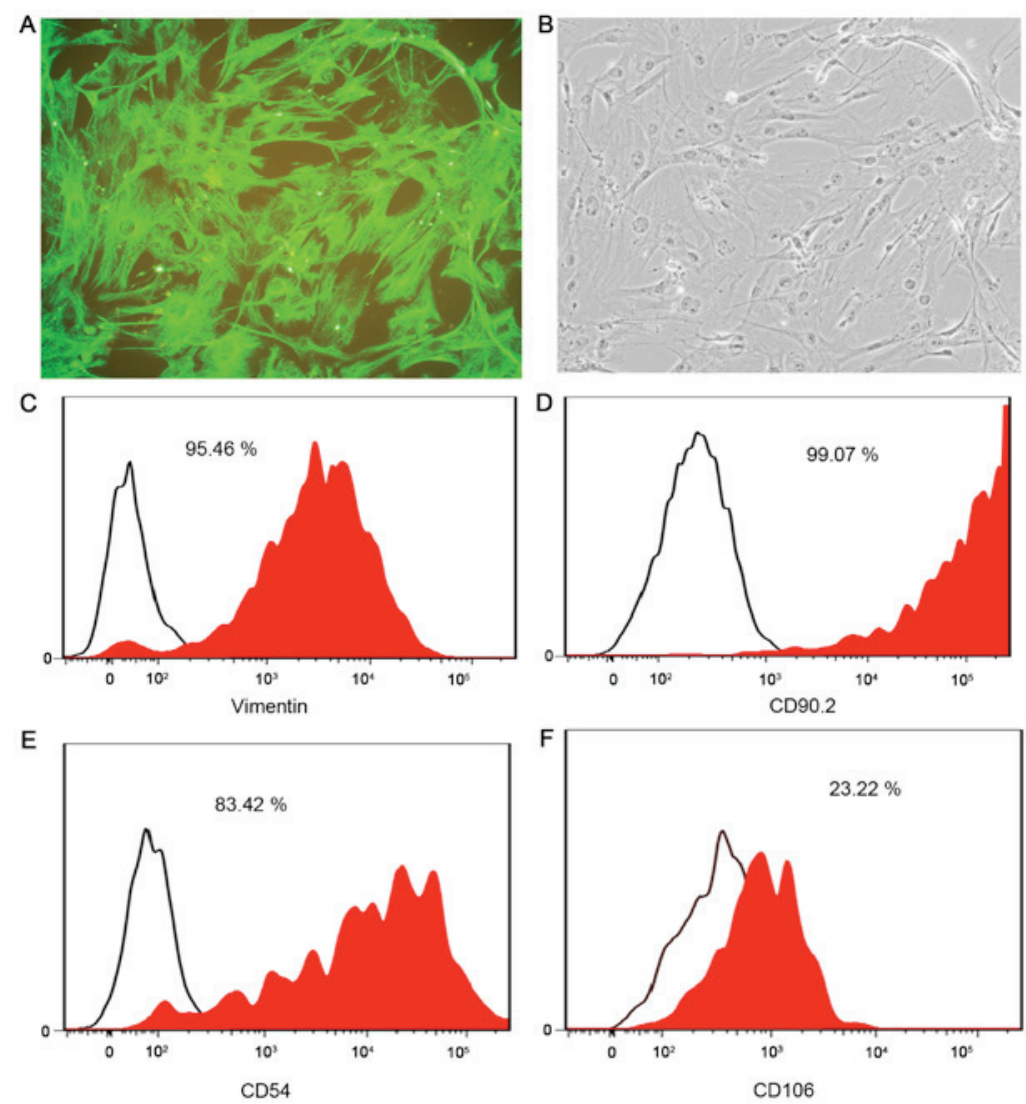

Figure 3. Purity analysis of SFs. (A) Immunocytochemical staining of SFs with the vimentin antibody under a Nikon eclipse inverted fluorescence microscope. (B) The contrasting cells did not stain. (C) Flow cytometric analysis indicating that vimentin labeled 95\% of SFs. (D) The marker CD90.2 labeled 99\% of SFs. (E) The marker CD54 labeled 83\% of SFs. (F) Only 23\% of CD106-positive SFs were detected. SFs, synovial fibroblasts; CD, cluster of differentiation.

\section{Discussion}

The methods applied for the primary culture of mouse SFs included tissue block culture and pancreatic enzyme and collagenase digestion (16-18). All these methods, however, have apparent shortcomings. The methods of tissue block culture are relatively easy, but the blocks are small and cannot be evenly distributed in the culture flasks. The majority of the cells grow around the blocks. Other challenges associated with the tissue block culture include blocks adhesive difficulty, a long growth period of the primary generation, a low survival rate and a small number of cells. The limitations of the pancreatic enzyme digestion method are that this technique cannot effectively digest and separate the fibrous tissues, impeding the dissociation of synovial cells. During filtering, the cells can block the filter pores. Therefore, a low cell number is obtained. The type II collagenase was initially used in combination with pancreatic enzyme digestion, as reported previously (19). This method, however, was found to be ineffective. A small quantity of cells with a low purity was obtained, and 15-20 days were required for the first passage.

Type IV collagenase can effectively digest and separate the fibrous tissues and intercellular matrix. The fibrous tissues are digested into filamentous fibers, and the cells are completely dissociated. The method only required one type of enzyme and no filtration process was required, thus, simplifying the experimental procedure and reducing cell loss. The concentration of type IV collagenase was increased gradually from $0.1 \%$, and $0.5 \%$ was found to be the most effective concentration. It is possible that a higher concentration would provide an improved effect. For example, a concentration of $1 \%$ may be superior to a concentration of $0.5 \%$. However, the concentration of type IV collagenase was not further increased in this experiment. Following digestion by type IV collagenase, the samples were vortexed vigorously for $1.5 \mathrm{~min}$ to release cells. A poor effect was observed when the vortex was $<1 \mathrm{~min}$. Vortex for $1.5 \mathrm{~min}$ was found to be equivalent to vortex for $3 \mathrm{~min}$. Therefore, the present study recommends $1.5 \mathrm{~min}$.

The synovium between the knee joints was also used for the culture. However, the purity of SFs was found to be low, potentially due to the small amount of synovial tissue between the knee joints or possibly due to the presence of cartilage resulting in a mixture of cartilage cells. In comparison to the synovium between the knee joints, a large amount of synovium was present around the hip joints (the greatest amount of dissociated synovium was present in the hip joints), and it was easy to excise the connective tissues under the stereomicroscope. Therefore, the synovium around the hip joints was mainly used for the culture. On day 3 after isolation, a large quantity of SFs was observed. The cells were trypsinized when confluence had reached $90-100 \%$ of the plate and they were transferred to $75-\mathrm{cm}^{2}$ flask (split 1:2) on days 6-8. A maximum number of synovial macrophages was observed at this time. Due to the firm adhesive of the synovial macrophages, the cells could not be easily detached by digestion with $0.25 \%$ pancreatic enzyme. Therefore, the cells were purified during 
the passage. After 3 passages, the majority of the macrophages were eliminated.

Vimentin is a type III intermediate filament protein that is expressed in mesenchymal cells. Due to this, vimentin is often used as a marker of SFs. CD90.2 is a $25-37 \mathrm{kDa}$ heavily N-glycosylated, glycophosphatidylinositol-anchored conserved cell surface protein with a single V-like immunoglobulin domain, originally discovered as a thymocyte antigen. It is initially described as SFs-specific, and positive identification of SFs was attempted using the antibody CD90.2 $(20,21)$. ICAM-1, also known as CD54, is a transmembrane protein possessing an amino-terminus extracellular domain, a single transmembrane domain and a carboxy-terminus cytoplasmic domain. VCAM-1, or CD106, is a protein that in humans is encoded by the VCAM1 gene. CD106 and CD54 belong to the immunoglobulin family of adhesion molecules, and they present on the SFs surface. Therefore, these four antibodies were selected in the present study to identify the purity of SFs.

In conclusion, the protocol described herein for the primary culture of murine SFs provides millions of primary cells per experiment, permitting experimentation in biochemistry and cellular biology investigations on the characteristics of SFs. Therefore, this method is a simple and effective way to obtain large quantities of murine SFs.

\section{Acknowledgements}

The authors would like to acknowledge the National Natural Science Fund of China (grant no. 81172875).

\section{References}

1. Emery P, Breedveld FC, Dougados M, Kalden JR, Schiff MH and Smolen JS: Early referral recommendation for newly diagnosed rheumatoid arthritis: Evidence based development of a clinical guide. Ann Rheum Dis 61: 290-297, 2002.

2. Dougados M, Devauchelle-Pensec V, Ferlet JF, Jousse-Joulin S, D'Agostino MA, Backhaus $M$, Bentin J, Chalès $G$, Chary-Valckenaere I, Conaghan P, et al: The ability of synovitis to predict structural damage in rheumatoid arthritis: a comparative study between clinical examination and ultrasound. Ann Rheum Dis 72: 665-671, 2013.

3. Bartok B and Firestein GS: Fibroblast-like synoviocytes: Key effector cells in rheumatoid arthritis. Immunol Rev 233: 233-255, 2010.

4. Neumann E, Lefèvre S, Zimmermann B, Gay S and Müller-Ladner U: Rheumatoid arthritis progression mediated by activated synovial fibroblasts. Trends Mol Med 16: 458-468, 2010.

5. Lefèvre S, Knedla A, Tennie C, Kampmann A, Wunrau C, Dinser R, Korb A, Schnäker EM, Tarner IH, Robbins PD, et al: Synovial fibroblasts spread rheumatoid arthritis to unaffected joints. Nat Med 15: 1414-1420, 2009.

6. Karouzakis E, Gay RE, Gay S and Neidhart M: Epigenetic control in rheumatoid arthritis synovial fibroblasts. Nat Rev Rheumatol 5: 266-272, 2009.
7. Noss EH and Brenner MB: The role and therapeutic implications of fibroblast-like synoviocytes in inflammation and cartilage erosion in rheumatoid arthritis. Immunol Rev 223: 252-270, 2008.

8. Perlman $\mathrm{H}$ and Pope RM: The synovial lining micromass system: Toward rheumatoid arthritis in a dish? Arthritis Rheum 62: 643-646, 2010.

9. Müller-Ladner U, Ospelt C, Gay S, Distler O and Pap T: Cells of the synovium in rheumatoid arthritis. Synovial fibroblasts. Arthritis Res Ther 9: 223, 2007.

10. Miao CG, Huang C, Huang Y, Yang YY, He X, Zhang L, Lv XW, Jin Y and Li J: MeCP2 modulates the canonical Wnt pathway activation by targeting SFRP4 in rheumatoid arthritis fibroblast-like synoviocytes in rats. Cell Signal 25: 598-608, 2013.

11. Pillinger MH, Dinsell V, Apsel B, Tolani SN, Marjanovic N, Chan ES, Gomez P, Clancy R, Chang LF and Abramson SB: Regulation of metalloproteinases and NF-kappaB activation in rabbit synovial fibroblasts via $\mathrm{E}$ prostaglandins and Erk: Contrasting effects of nabumetone and 6MNA. Br J Pharmacol 142: 973-982, 2004.

12. Tao K, Zeng H, Xiao DM, Xiong A, Weng J and Kang B: Influences of IL-6R antibody on PMMA bone cement-mediated expression of OPG and RANKL in synovial fibroblasts. J Huazhong Univ Sci Technolog Med Sci 34: 241-246, 2014.

13. Fiorito S, Magrini L, Adrey J, Mailhé D and Brouty-Boyé D: Inflammatory status and cartilage regenerative potential of synovial fibroblasts from patients with osteoarthritis and chondropathy. Rheumatology (Oxford) 44: 164-171, 2005.

14. Pierer M, Brentano F, Rethage J, Wagner U, Hantzschel H, Gay RE, Gay S and Kyburz D: The TNF superfamily member LIGHT contributes to survival and activation of synovial fibroblasts in rheumatoid arthritis. Rheumatology (Oxford) 46: 1063-1070, 2007.

15. Scian R, Barrionuevo P, Rodriguez AM, Arriola Benitez PC, García Samartino C, Fossati CA, Giambartolomei GH and Delpino MV: Brucella abortus invasion of synoviocytes inhibits apoptosis and induces bone resorption through RANKL expression. Infect Immun 81: 1940-1951, 2013

16. Nikitopoulou I, Oikonomou N, Karouzakis E, Sevastou I, Nikolaidou-Katsaridou N, Zhao Z, Mersinias V, Armaka M, $\mathrm{Xu} \mathrm{Y}$, Masu M, et al: Autotaxin expression from synovial fibroblasts is essential for the pathogenesis of modeled arthritis. J Exp Med 209: 925-933, 2012.

17. Ohki E, Suzuki M, Aoe T, Ikawa Y, Negishi E and Ueno K: Expression of histamine $\mathrm{H} 4$ receptor in synovial cells from rheumatoid arthritic patients. Biol Pharm Bull 30: 2217-2220, 2007.

18. Brühl H, Mack M, Niedermeier M, Lochbaum D, Schölmerich J and Straub RH: Functional expression of the chemokine receptor CCR7 on fibroblast-like synoviocytes. Rheumatology (Oxford) 47: 1771-1774, 2008.

19. Zimmermann T, Kunisch E, Pfeiffer R, Hirth A, Stahl HD, Sack U, Laube A, Liesaus E, Roth A, Palombo-Kinne E, et al: Isolation and characterization of rheumatoid arthritis synovial fibroblasts from primary culture--primary culture cells markedly differ from fourth-passage cells. Arthritis Res 3: 72-76, 2001.

20. Saalbach A, Aneregg U, Bruns M, Schnabel E, Herrmann K and Haustein UF: Novel fibroblast-specific monoclonal antibodies: Properties and specificities. J Invest Dermatol 106: 1314-1319, 1996.

21. Rosengren S, Boyle DL and Firestein GS: Acquisition, culture, and phenotyping of synovial fibroblasts. Methods Mol Med 135: 365-375, 2007. 